\title{
Germanica
}

GERMANICA $7 \mid 1990$

Grenze und Entgrenzung

\section{Grenze in Entgrenzung. Bataille über Hegel}

\section{Wolfram Malte Fues}

\section{OpenEdition}

Journals

Édition électronique

URL : http://journals.openedition.org/germanica/2487

DOI : 10.4000/germanica. 2487

ISSN : 2107-0784

\section{Éditeur}

Université de Lille

\section{Édition imprimée}

Date de publication : 30 juin 1990

Pagination : 27-36

ISSN : 0984-2632

\section{Référence électronique}

Wolfram Malte Fues, « Grenze in Entgrenzung. Bataille über Hegel », Germanica [Online], 7 | 1990,

Online erschienen am: 17 Juli 2014, abgerufen am 06 Oktober 2020. URL : http://

journals.openedition.org/germanica/2487 ; DOI : https://doi.org/10.4000/germanica.2487

Ce document a été généré automatiquement le 6 octobre 2020.

(c) Tous droits réservés 


\title{
Grenze in Entgrenzung. Bataille über Hegel
}

\author{
Wolfram Malte Fues
}

1 «Le soleil ni la mort ne se peuvent regarder fixement », La Rochefoucauld.

2 «Das Licht nämlich als Naturelement ist das Manifestierende; ohne dass wir es selber sehen, macht es die erhellten, beschienenen Gegenstände sichtbar. Den gleichen Charakter des Manifestierens hat der Geist, das freie Licht des Bewusstseins, das Wissen... und das Erkennen.

Das Positive und Negative ist dasselbe. Dieser Ausdruck gehört der äusseren Reflexion an, insofern sie mit diesen beiden Bestimmungen eine Vergleichung anstellt. Es ist aber nicht eine äussere Vergleichung, welche zwischen denselben... anzustellen ist, sondern sie sind an ihnen selbst $\mathrm{zu}$ betrachten, d.h. es ist $\mathrm{zu}$ betrachten, was ihre eigene Reflexion ist. An dieser aber hat es sich gezeigt, dass jedes wesentlich das Scheinen seiner im Anderen und selbst das Setzen seiner als des Anderen ist. ... So gilt, um ein Bespiel von dem fixen Gegensatze dieser Reflexionsbestimmungen anzuführen, das Licht überhaupt für das nur Positive, die Finsternis aber für das nur Negative. Aber das Licht hat in seiner unendlichen Expansion und der Kraft seiner abschliessenden und belebenden Wirksamkeit wesentlich die Natur absoluter Negativität. Die Finsternis dagegen als Unmannigfaltiges oder der sich nicht selbst in sich unterscheidende Schoss der Erzeugung ist das einfache mit sich Identische, das Positive.» G.W.F. Hegel

«De la fermeté du désespoir, éprouver le plaisir lent, la rigueur décisive, être dur et plutôt garant de la mort que victime. ... Le désespoir est simple : c'est l'absence d'espoir, de tout leurre. C'est l'état d'étendues désertes et - je puis l'imaginer - du soleil.

... Plaisanterie de vouloir être un homme au fil de l'eau, sans jamais se traquer, forcer les derniers retranchements : c'est se faire le complice de l'inertie. L'étrange est qu'en se dérobant l'on ne voit pas la responsabilité assumée: aucune ne peut accabler davantage, c'est le péché inexpiable, la possibilité une fois entrevue de l'abandonner pour les lentilles d'une vie quelconque. La possibilité est muette, elle ne menace ni 
maudit, mais celui qui, craignant de mourir, lui, la laisse mourir, est comme un nuage décevant une attente de soleil. » Georges Bataille

Hoffnungslosigkeit - so Bataille in «L'expérience intérieure» - ist eine einfache Erfahrung: nichts weiter als die Abwesenheit aller Hoffnung, jeden Köders, der zugleich lockt und täuscht, der nicht anziehen kann, ohne zu verlocken. Ihr entspricht die unschlüssige Lust, die entschiedene Strenge, eher Garant - und Meister? - des Todes zu sein als dessen Opfer, die zweifache und zwiespältige Empfindung, zu der sich die Hoffnungslosigkeit entschliesst und in der sie sich einschliesst. "Désespoir » meint wörtlich genommen nicht «Hoffnungslosigkeit». Es stellt keinen Zustand fest, sondern beschreibt einen Prozess, den Vorgang der «Ent-Hoffnung». In der Festigkeit, die den Tod nicht aus den Augen lässt, unschlüssig, ob sie an ihn rühren soll, kommt eine Bewegung zum Abschluss, die alles Lebensmögliche durchlaufen und beiseite gelassen hat, so fern es auch von ihrer endgültigen Einigkeit mit sich selbst abzulenken und wegzulocken vermag. Dieser Schluss wiegt schwer, weil er den Weg der Ent-Hoffnung enthält, und leicht, weil alle Vermittlung, jeder begrenzende und entgrenzende Uebergang in ihm aufgebraucht ist und nichts bleibt als sich ergebende Unmittelbarkeit, einfaches, wunschlos glückliches Unglück. Sein, reines Bei-sichSein ohne alle Selbst-Bestimmung: Wüsten im Endstand ihrer Unendlichkeit oder - was dasselbe zu sein scheint - eine Sonne, die überall im Zenit steht.

Das Licht der Natur und das Licht der Vernunft sind Hegel zufolge vom gleichen Charakter, sie bestimmen einander wechselseitig, und jedes wird in dieser Weise die von seinem Anderen bestimmte Bestimmtheit. In solcher Reflexion, durch die sich die moderne Vernunft die Lichtmetaphorik methodisch einverleibt, gilt das Licht nicht mehr als Metapher, sondern als das Metaphorische selbst, als das Medium, in dem die Uebertragung theoretische Bedeutung annimmt. Natur und Vernunft, Wissen und Manifestieren bringen in ihrer Reflexion ihr Erscheinen selbst zu einer Erscheinung, in der es sich von seiner blossen Reflexform, seiner dem wechselseitigen Anschein dienenden Unsichtbarkeit befreit. Auch im Reich des Geistes geht, folgt man Hegel, die Sonne nicht unter. Dieselbe Sonne, in deren Licht sich nach Bataille der Prozess der Ent-Hoffnung, der De-Realisierung erschöpft? Oder ist das eine andere, eine dunkle Sonne vielleicht? Und wenn ja: Welche Mitte, welche Reflexion jenseits der Erschöpfung aller Reflexion, vermittelt dann zwischen ihnen? Die Uebertretung statt der Uebertragung?

Die Fährten der von der Hoffnung ausgelegten Realität führen deren Subjekt letzten Endes zum "plaisir lent ", jener trägen, unschlüssigen Lust an der Gegenwart des Todes, die ihn im Licht der Hoffnungslosigkeit garantiert, aber nicht aktualisiert, aufziehen lässt wie eine schon ins Unendliche erstreckte Nacht. In der Perspektive dieses Erlebnisses muss jeder Versuch, ein Leben in üblicher Stromlinienförmigkeit verfliessen $\mathrm{zu}$ lassen, ohne es jemals bis in seine letzten Ausflüchte und Verschanzungen $\mathrm{zu}$ verfolgen, um es daraus zu vertreiben, wie eine "plaisanterie ", wie ein begnügungsfrohes Bündnis mit einer existentiellen Trägheit aussehen, die nicht mehr Unschlüssigkeit angesichts ausser ster Selbsterhellung, sondern die Feigheit ist, sich an dem Reflexionsprozess zu beteiligen, aus dem deren Licht blendend aufgeht. «Weder die Sonne noch den Tod kann man fest ins Auge fassen.» Aber eben hierin und hierdurch besteht der Blick, auf den es ankommt, weil alles Sehen bei ihm ankommt.

7 Die Subjekte zivilisierter Gesellschaften haben meist die "plaisanterie » zum Grund ihrer Lebensgeschichte. Sie werden dazu getrieben, sich treiben zu lassen, und erleben 
ihr Schicksal keineswegs als den faden Scherz existentieller Unfähigkeit, sondern als Treibjagd nach einem Platz an der Sonne, auf der jeder Jäger und Gejagter zugleich ist. Das Subjekt, wie Bataille es auffasst, das su-jet, weiss sich als Gegenstand dieser Bewegung, weiss sie zugleich als den Prozess seines Selbstbewusstseins und sich so mit ihr eins und einig. Es ist ihr unterworfen, und was es von sich weiss, hat die Form dieser Unterwerfung, die es sich in ihr vorwirft, während es sich allein in diesem Vorwurf sein Selbst zu entwerfen vermag. Die erste und allgemeine Bestimmung des su-jet ist das pro-jet, sind die Projekte, in die es von der Hoffnung gelockt wird, sich durch sie gegen seine Subjektivität zu erheben, sie als seine Substanz darin aufzuheben und zugleich sich als von seiner Subjektivität befreites, souveränes Subjekt aufzuheben, zu bewähren und zu erhalten. Letztlich dieser Wunsch nach Befreiung, das Begehren, auch um den Preis des subjektiven Todes Herr über die eigene Unterwürfigkeit zu werden - « le désir » nennt es Bataille -, lockt das Subjekt auf den Weg der Projekte, in die Fata Morgana einer "expérience extérieure", wie sie sich im Sinn der Uebertragung nennen lässt, die ihm gegenständliche, begegnende Bestimmtheit und das Ende ihrer Herrschaft zugleich brächte. Kein Projekt aber reicht über seinen Ursprung hinaus. Vergegenständlichung und Identifikation, der Prozess der Selbstrealisation und sein Rezess auf einfaches BeisichSein, Selbstbestimmungsvorstellung und Todesbegehren formen jedes Projekt des Subjekts nach dem Widerspruch, den es bildet und durch den es sich entwickelt, werfen das Begehren auf sich selbst zurück und machen es der Hoffnung Untertan, im nächsten und immer wieder nächsten Projekt das zu finden, was es in diesem vergebens gesucht hat. Die übliche Lebenserfahrung des Subjekts verläuft (sich) in diesem Hin und Wider der " expérience extérieure ", im Pendelschlag zwischen pro-jet und re-jet.

Das Positive und Negative ist dasselbe. So lautet die lebenslängliche Erfahrung des Subjekts. Das Licht, in dem es seiner ansichtig wird, erscheint ihm zunächst als das Positive weil Positionierende, die Finsternis des schlichten Bei-sich-Seins und Bleibens dagegen als das Negative weil ohnmächtig Bestimmungslose. Während es sich auf den Schein dieses Erscheinens einlässt, vergeht es als die Einfachheit, in der es sich vorfindet und deren letztendlich einigende Einheit es in aller fehlschlagenden Hoffnung unbeirrbar verfolgt. In Konsequenz dieser Fehlschläge erlebt es das Licht der Reflexion, in dem sich seine Selbstbestimmung manifestiert, als das Negative, Verneinende, Bestimmtheit nach Bestimmtheit Schritt für Schritt Vernichtende, die Finsternis des blossen Bei-sich-Seins jedoch als das in allem sich auflösenden Anschein manifest bleibende Positive. Das Positive und das Negative, Licht und Finsternis tauschen im endlosen Kreuzschritt der Reflexion die Plätze und machen das Subjekt zum sujet eines Wechselspiels, in dem es, zwischen projet und rejet, Todesfurcht und Todeswunsch hin und her geworfen, den Tod nicht findet, sondern irgendann dessen zufällige Fundsache wird.

In derselben Reflexion vollzieht sich, folgt man Hegel, der Uebergang vom natürlichen Manifestieren zum erkennenden. Das Metaphorische in der Reflexionsform, den sich wiederholenden und in der Wiederholung verändernden Spiegelungen, befreit das Licht, in dessen Schein Sein sich zum Dasein, Berührung und Empfindung sich zu Erörterung und Bestimmung distanzieren und differenzieren, von seiner natürlichen Eindeutigkeit und Einlinigkeit, aber noch nicht von den Ansprüchen des Selbstbewusstseins, die sich ihm unterwerfen, um es sich zu unterwerfen. Derartige Freiheit gewinnt, wie Batailles Dialektik des Subjekts gezeigt hat, die Reflexion selbst ihm ab, seine Hoffnungen erfüllend, indem sie sie enttäuscht, sie enttäuschend, indem 
sie ihnen demnächst und immer wieder demnächst Erfüllung verspricht. Diese Freiheit entfaltet sich in die mannigfaltige Einfachheit des absoluten Wissens, die universale Metaphorik des Gegenständlichen überhaupt, die in allen ihren Gegenständen nur sich, in allen Uebertragungen nur das Uebertragen selbst zum Gegenstand hat: «Der Begriff oder die wahrhafte Unendlichkeit überhaupt und damit die des Geistes ist, dass die Schranke als Schranke für ihn sei, dass er sich in seiner Allgemeinheit bestimme, d.i. sich in eine Schranke setze, aber dass sie als ein Schein sei; er ist dies, ewig sich diesen Schein zu setzen, die Endlosigkeit nur als ein Scheinen in ihm zu haben.» ${ }^{1}$

Führt dann nicht ein ununterbrochener Weg von der Subjektivität zu ihrer Liquidation, zu ihrer Auflösung im umfassenden Zenit einer Sonne, die alle Reflexion in reines Scheinen verflüssigt hat und in dessen Glanz einen Tod stellt, der nichts Gegenständliches und nichts Identisches mehr hat, sondern nur noch die Gegenwart entschlussfordernder Andersheit? Ist es demzufolge nicht geradezu notwendig, «être un homme au fil de l'eau », sich von der Metaphorik der Reflexion durch die Licht und Schattenseiten des üblichen Lebens ohne Eigensinn tragen zu lassen? Welche Aussicht soll in diesem unendlichem Spiegelkabinett und seinem sich von selbst organisierenden Gestaltenspiel noch offen sein, welche darin noch stumme, noch nicht manifeste Möglichkeit vermag noch in den Blick zu kommen und warum soll es eine unverzeihliche Sünde sein, gerade sie zu übersehen?

11 Wie das Subjekt an der unermüdlichen Reflexion seiner Hoffnung auf die Reflexion zugrundegeht, hat Batailles negative Dialektik des Subjekts gezeigt. "Le désespoir ", die reine Negativität der sich zu sich beschliessenden Reflexion, in der alle ihre Projekte aufgeschlossen und in die blosse Bewegung des Projizierens eingeschlossen sind, der Zustand des Subjekts unter einer Sonne, die keinen Schatten mehr duldet, entspricht dem, was Hegel «Begriff», nennt, die Unendlichkeit, für die auch die letztendlichen Fluchten und Verschanzungen des Daseins nur der Schein sind, in dem sie vergehen. Le dés-espoir - der Be-griff. Die Entsprechung ist zugleich ein Widerspruch. Denn der Begriff, wie sein Name sagt, entschränkt den Prozess der Reflexion nicht nur in seine reine Selbstbewegung, sein bloss negativ unendliches Beisich-Sein, sondern stellt darin die Schranken und Dämme, die Fluchtorte und Verschanzungen des Projekts Hoffnung zugleich wieder her, setzt sie in den Schein, der sie zersetzt, und ersetzt sie aus ihm und durch ihn. Diese Einheit von Destruktion und Konstruktion, die eigentlich de-konstruktive Methode der modernen Vernunft, konkretisiert das freie Licht des Geistes zum System, zu wahrhafter, sich in bestimmter Endlichkeit bewährender Unendlichkeit. In ihr bescheidet es sich zu einer Intelligenz, die aus dem Licht ihrer Einsicht in sich selbst ihr eigentümliches Schattenreich entwirft und selbstironisch - spekulativ - darauf verzichtet, die wirklich unendliche Gewalt, die sie über sich errungen hat, an die Wahl eines eigenen Todes in ihrem äussersten Anderen $\mathrm{zu}$ wenden und ihn stattdessen über die Residenzen ihres wahrhaften Scheins als Statthalter ihrer Resignation systematisch verteilt. Sein und Schein sind dasselbe geworden und erhalten sich in der sich an ihrer jeweiligen Endlichkeit bewährenden Unendlichkeit ihres Werdens, im S e t z e n des Scheins wie im Setzen des S c h e i n s. Fazit: «Das Dialektische führt sich selbst herbei, und darin liegt dann das Spekulative, insofern das Positive des Dialektischen aufgefasst wird.»². Hier, an diesem Punkt endet die reflexive Verbindung, die Batailles Texte - immer unter Vorbehalt des Spekulativen - mit denen Hegels eingehen. Der Vorbehalt isoliert und radikalisiert sich jetzt zur unbedingten Negation. "Hegel, je l'imagine, toucha l'extrême. Il était jeune encore et crut devenir fou. J'imagine même qu'il élaborait le 
système pour échapper (chaque sorte de conquête, sans doute, est le fait d'un homme fuyant une menace). Pour finir, Hegel arrive à la satisfaction, tourne le dos à l'extrême. ... Hegel gagna, vivant, le salut, tua la supplication, se mutila. ... Mais avant de se mutiler, sans doute il a touché l'extrême, connu la supplication : sa mémoire le ramène à l'abîme aperçu, pour l'annuler! Le système est l'annulation. $»^{3}$ Hegel hat mit der Stabilisierung der Hoffnung gegen den und in dem Prozess der " désespoir ", mit der Neutralisierung des Dialektischen durch das Spekulative in der Erfindung des Systems, die äusserste, unverzeihliche Sünde begangen. Er hat, was menschenmöglich ist, «le possible de l'être ", in seiner letzten Bestimmtheit, in der es sich als Bestimmen selbst vernichtet, zu Gesicht bekommen und ihm den Rücken zugewandt. Er hat sich von der letzten Sequenz des Negativen zurückgezogen, in der es als Folgerungsform des wirklich Möglichen endet und die sich deshalb nicht erzwingen, sondern nur erwarten lässt, sie in den Prozess, der sie vorbereitet, zurückgebunden, die "supplication » durch die «satisfaction » verdrängt. Die Angst vor der Todesangst im Begehren des Todes hat ihn aus dem Anblick eines Sonnenlichts vertrieben, dem sich die Finsternis so unmittelbar amalgamiert, dass nicht einmal der Begriff Unterschied und Einheit zwischen ihnen auszumachen vermag. Er sieht, folgt man Bataille, diese Sonne nur noch durch Linsen an, die mit Hoffnung gefärbt sind, und vergibt die Erfahrung des ungeschützten Auges für das Linsengericht irgendeines üblichen Lebenslaufes, «pour les lentilles d'une vie quelconque ». So täuscht er sich und andere über die wahre Unendlichkeit im Ende der Wahrhaftigkeit hinweg wie eine Wolke, die sich zwischen die Sonne und ihre subjektive Erwartung schiebt, «comme un nuage décevant une attente de soleil».

" La possibilité », das Menschen Mögliche, droht und verdammt nicht. Es ruft und lockt stumm und zwiespätlig, ins Leben der Projekte und in dessen Tod. Laut und bedrohlich wird es erst für eine Subjektivität, die sich an der Erfüllung ihrer ersten Hoffnung in deren letzter De-Realisierung nicht beteiligt, inert wie ein chemisch träger Stoff im System der Elemente gegen die elementare EntSchliessung des Systems. « Sortir par un projet du domaine du projet» ${ }^{4}$, fordert Bataille. Aber dieses letzte, äusserste Projekt, das die Sphäre des Projektier ens überhaupt hinter sich zu lassen sucht, ist mit den Mitteln der Reflexion nicht zu verwirklichen, sondern einzig mit deren Zerstörung, im Bruch mit der Selbst-Bewegung des Subjekts. Wo die Uebertragung versagt, muss die Uebertretung einspringen. Zwischen dem Reich der " possibilité » und seiner überall und immer gegenwärtigen Zukunft - «l'extrême du possible» - liegt " une extrême déchirure, si profonde que seul le silence de l'extase lui répond. ... Le désir, la poésie, le rire, font incessamment glisser la vie dans le sens contraire, allant du connu à l'inconnu. $»^{5}$. Vielleicht auf den rückhaltlosen Stufen phonologisch-anagrammatischer Metonymie : le rire - la déchirure - le déchirire - déchirer - déchiffrer?

Grenze und Entgrenzung: auf diesen Nenner lässt sich Hegels und Batailles gemeinsame Auffassung von dem Prozess bringen, den das Subjekt der Moderne seit Descartes' «Meditationes» um sein Selbst gegen sich führt, den es gewinnt, indem es sich verliert, und den es verliert, indem es ein privates Schicksal gewinnt. Es muss die Hauptklage um sein Projektieren überhaupt, könnte man mit Hegel sagen, um die Erfüllung und Auflösung seiner Subjektivität in deren vollständiger Manifestation, verlieren, damit die Nebenklage um ein privates Schicksal, einen aus besonderen Projekten besonders verbundenen Lebenslauf, Erfolg hat. Der allgemeine Prozess geht somit über es hinweg, befreit sich von ihm zu seiner nur ihn manifestierenden Reflexion in sich, zu einer Dialektik der Grenze als Entgrenzung und Begrenzung. Hier widerspricht Bataille. Das 
moderne Subjekt erschöpft sich ihm zufolge nicht im zweideutigen Kampf mit dieser Dialektik, es erschöpft nur eine Subjektivität, die von der Hoffnung zur Aktion getrieben wird, sie vermöge ihrem zwischen projet und rejet zwiespältigen sujet die Satisfaktion seiner verlustfreien Einigung mit sich selbst zu leisten. Die Befreiung vom Anspruch dieser Subjektivität erlebt das Subjekt als Freisetzung von seiner ursprünglichen und anfänglichen Form, als Uebergang von der Hoffnung zur Hoffnungslosigkeit, zu jenem " désespoir ", der die "expérience intérieure » eröffnet. Hegel setzt an diesem Uebergang das System spekulativ gegen das Subjekt ein, indem er es in seiner ursprünglichen und anfänglichen Subjektivität restauriert und die Selbstdarstellung der Dialektik selbst zum Medium der Restauration macht. Diesem Versuch, der "possibilité de l'être ", der das Wirkliche erschöpfenden Dialektik des Möglichen, die Spitze abzubrechen, durch den sie mit ihrer eigenen Entstehungsgeschichte in permanenter Wiederholung konkretisiert wird, verweigert Bataille die Gefolgschaft. Die Geschichte der Vernunft als diejenige des modernen Subjekts ist für ihn ihrer Form und ihrer Methode nach mit Hegels Auffassung ihrer konstitutiven Negativität abgeschlossen. Wer sich durch sie diesem Schluss aussetzt, der unschlüssigen Lust auf ihren Tod, muss ihre zukünftige Gestalt jenseits von ihr aufsuchen, im Gelächter über und im Riss durch ihr Konzept des Menschen. Grenze und Entgrenzung verschwinden im Abgrund - und Ungrund - zwischen der Sonne, in deren Licht das Subjekt rein und negativ vollkommen bei sich ist, und dem äussersten Anderssein, dem Tod, zu dem sich diese Negativität in ihrer letzten Negation entschliesst ${ }^{6}$.

In Georges Batailles « Roman inédit: Ma Mère », dessen Text von Anfang an über sehr erörterungswürdige moralische und psychologische Reflexionen hinweg unaufhaltsam dem Inzerst zu gleitet, heisst es : «Il me semblait que l'impureté monstrueuse de ma mère - et que la mienne, aussi répugnante - criaient au ciel et qu'elles étaient semblables à Dieu, en ce que seules les parfaites ténèbres sont semblables à la lumière. Je me souvenais de la phrase lapidaire de La Rochefoucauld : "Le soleil ni la mort ne se peuvent regarder fixement". » noch der Tod können sich unverwandt ansehen. $"^{8}$. So falsch für den Text La Rochefoucaulds, so richtig im Kontext Batailles. Auf diesen Blick haben es seine Texte überall abgesehen.

\section{NOTES}

1. - G.W.F. Hegel, Fragment zur Philosophie des Geistes [1822ff.]; Werke in 20 Bänden, Frankfurt 1970, Bd. 11, S. 531.

2. - Hegel an Niethammer am 24. März 1812; Briefe von und an Hegel, hrsg. von Johannes Hoffmeister, 3., durchges. Aufl. Hamburg 1969, Bd. 1, S. 398.

3. - Georges Bataille, L'expérience intérieure; Cuures Complètes; Bd. V, Paris 1973, S. 56.

4. - Bataille a.a.O., S. 60.

5. - Bataille a.a.O., S. 128 und S. $129 f$. 
6. - Allerdings: «Die erste Bestimmtheit aber und Negation in sich des Absoluten kann nicht die freie Selbstbestimmung des Geistes als Geistes, sondern selber nur die unmittelbare Negation sein. Die unmittelbare und dadurch natürliche Negation in ihrer umfassendsten Weise ist der Tod. ... Der Tod hat eine gedoppelte Bedeutung: einmal ist er das selbst unmittelbare Vergehen des Natürlichen, das andere Mal der Tod des nur Natürlichen und dadurch die Geburt eines höheren, des Geistigen, welchem das bloss Natürliche in der Weise abstirbt, dass der Geist dies Moment als zu seinem Wesen gehörig an sich selbst hat» (Hegel, Vorlesungen über die Aesthetik, a.a.O. Bd. 13, S. 450 u.f.). Von hier her wäre umgekehrt mit Hegel über Bataille zu reden.

7. - Ma Mère, roman inédit, éd. Jean-Jacques Pauvert, Paris, 1966, S. 56.

8. - Georges Bataille, Das obszöne Werk, hrsg. und übers, von Marion Luckow, Hamburg, 1972, S. 101. 